\title{
Editorial
}

\section{Can an iPS cell secure its genomic fidelity?}

\author{
R Sarig ${ }^{1}$ and V Rotter ${ }^{\star 2}$ \\ Cell Death and Differentiation (2011) 18, 743-744; doi:10.1038/cdd.2011.14
}

The hallmark of stem cells is their capacity to maintain a stringent mechanism to ensure their genomic integrity. This is particularly crucial for embryonic stem (ES) cells, which hold the potential to give rise to an entire organism, including its germ cells. In contrast to mutations occurring in mature somatic cells, mutations in ES cells may be endowed to various cell types in the organism, and may be passed on to its progeny. Indeed, mutation frequency in ES cells is much lower compared with somatic cells. ${ }^{1}$ To achieve this, ES cells are extremely sensitive to DNA damage and they readily undergo apoptosis or differentiation, resulting in sequestering of damaged cells from the pluripotent pool. ES cells lack a functional G1 checkpoint, enabling newly formed cells with DNA damage to enter another phase of DNA replication. Escalating the DNA damage leads to mutational burden and promotes apoptosis. In addition, ES cells differ from somatic cells in their capacity to repair damaged DNA. Somatic cells predominantly utilize error-prone nonhomologous end joining, which may promote accumulation of mutations, whereas ES cells predominantly utilized the high-fidelity homologymediated repair pathway, thereby minimizing the incurrence of mutations during the repair process. ${ }^{2,3}$

Somatic cells can be reprogrammed with a defined set of transcription factors to produce induced pluripotent stem (iPS) cells. ${ }^{4}$ Gene expression profiling, epigenetic signatures as well as differentiation potential demonstrated the close resemblance of iPS cells to ES cells. Therefore, it is possible to envision numerous therapeutic applications for iPS cells as an unlimited source of patient-specific cells and for the study of genetic diseases. However, the benefits of iPS cells may be hampered by safety concerns such as their tumorigenicity. As several reprogramming factors possess oncogenic activity (e.g., c-Myc, Klf4, Oct4, Lin28), known to induce genomic instability, the alerting oncogenic potential inherited by this technology should be kept in mind. Reprogramming, which entails the reversion of terminally differentiated cells into the ES state resembles closely the process of transformation. In both processes, somatic cells with limited proliferation capacity acquire indefinite self-renewal ability after extensive modifications, including profound changes to their epigenetic status. Reprogramming of somatic cells, which already carry defects in their tumor-suppressing machinery, may tilt their destiny towards malignant transformation rather than towards normal fate. p53, 'the guardian of the genome', is a major factor in preventing transformation and has a crucial role in tumor suppression. ${ }^{5}$ It was shown to be required for the maintenance of chromosomal integrity and stability in both somatic and ES cells. The p53 pathway is activated upon reprogramming, mainly to induce growth arrest and apoptosis of DNA-damaged cells. Inactivation of p53 results in enhanced efficiency of reprogramming. ${ }^{6}$ However, not surprisingly, this was accompanied by an enhanced tumorigenic potential of the reprogrammed cells. ${ }^{7}$ Therefore, for iPS cells to perform as authentic ES cells, it is inevitable for them to adopt the stringent regulation machinery used by ES cells to maintain their genomic integrity, and to prevent the accumulation of cells with chromosomal aberrations in the pool of reprogrammed cells.

It is critical to ensure that iPS cells reprogrammed by the conventional methods, using the traditional three (Oct4, Sox2 and Klf4) or four (Oct4, Sox2, Klf4 and $c-M y c)$ factors indeed possess the ability to eradicate chromosomal aberrations occurring along the process, and to verify genomic integrity during prolonged culture conditions.

Comparing the DNA damage response of iPS and ES cells, it was previously shown that human iPS cells acquire a similar response as ES cells do, resulting in loss of the G1/S checkpoint and a marked increase in radiosensitivity. They possess efficient double-strand break repair and high expression of DNA damage signaling and repair genes. ${ }^{8}$ Nevertheless, a comprehensive analysis of numerous human iPS cell clones revealed a substantial number of cell lines carrying chromosomal aberrations. Some of them originated from the parental somatic cells, some were acquired during early passages and some appeared only after prolonged culturing. ${ }^{9}$ Prolonged culturing of ES cells may also lead to chromosomal aberrations resulting in aneuploid cells with growth advantage that takes over the population. ${ }^{10}$ The fact that iPS cells may inherit chromosomal aberrations originating from their parental somatic cells, or acquire these changes during early passages suggest the performance of incompetent machinery required for a stringent selection of pristine cell populations during this process. This may be explained by the fact that the parental somatic cells do not possess the high-fidelity mechanism, typical for ES cells along the reprogramming process.

\footnotetext{
${ }^{1}$ Department of Biological Regulation, Weizmann Institute of Science, Rehovot, Israel and ${ }^{2}$ Department of Molecular Cell Biology, Weizmann Institute of Science, Rehovot, Israel

*Corresponding author: V Rotter, Department of Molecular Cell Biology, Weizmann Institute of Science, Rehovot 76100, Israel. Tel: + 97289344501 ;

Fax: + 9728934 4125; E-mail: varda.rotter@weizmann.ac.ll
} 
Although aneuploid karyotypes reflect gross chromosomal aberrations, the presence of a normal diploid karyotype in various previously analyzed iPS clones does not guarantee the absence of genomic alterations. In this issue, Pasi et al. ${ }^{11}$ tested whether reprogramming of somatic cells may result also in more subtle genomic aberrations than aneuploidy. In addition to the conventional methods using three or four factors to produce iPS cells, they reprogrammed primary mouse mammary cells into mammary stem cells by expression of $c$-Myc. Using array-based comparative genomic hybridization analysis, they demonstrate genomic alterations in reprogrammed cells that were associated with oncogeneinduced DNA replication stress. Despite the observed changes, the induced mammary stem cells were capable of properly repopulating mouse fat pads without initiating tumor formation, and the iPS cells were able to give rise to viable fertile mice, although with low efficiency. Thus, the authors suggest that the observed genomic aberrations may be of a transient nature and do not compromise the cells' function. ${ }^{11}$ It might be as well that the stringent conditions regulating the development of an entire embryo enable the selection of only cells with an intact genome, whereas cells carrying any fatal abnormalities are disposed of during either early or late stages. That may explain the low efficiency of this process. It should be mentioned that because of its high oncogenic potential, $c-M y c$ has lost its clinical therapeutic significance in the generation of iPS cells. As most studies, including the one by Pasi et al., ${ }^{11}$ analyzed genomic aberrations and functionality of iPS cells at early stages of their production, it might be that these alterations may be exacerbated during more prolonged culturing periods, and a population of cells gaining growth advantage will occupy most of the population. These transformed cells may be readily subjected to oncogenic transformation. In addition, newly acquired genomic alterations occurring in few cells endowed with growth advantage (which may not be detected using conventional methods) may occupy a pool of cells with intact genome that may be considered for the usage of various therapeutic applications.

The recently accumulated data, demonstrating genomic alterations in various iPS cell lines, stress the importance to evaluate the extent and nature of genomic instability of iPS cells. These aberrations may affect their differentiation capacity as well as enhance their tumorigenic potential, thus, resulting in the formation of cancer stem cells instead of normal pluripotent stem cells.

\section{Conflict of interest}

The authors declare no conflict of interest.

1. Stambrook PJ, Tichy ED. Adv Exp Med Biol 2010; 695: 59-75.

2. Hong $Y$ et al. Mutat Res 2007; 614: 48-55.

3. Tichy ED, Stambrook PJ. Exp Cell Res 2008; 314: 1929-1936.

4. Okita K, Yamanaka S. Exp Cell Res 2010; 316: 2565-2570.

5. Levine AJ, Oren M. Nature Reviews Cancer 2009; 9: 749-758.

6. Krizhanovsky V, Lowe SW. Nature 2009; 460: 1085-1086.

7. Sarig R et al. J Exp Med 2010; 207: 2127-2140.

8. Momcilovic $O$ et al. PLoS One 2010; 5: e13410.

9. Mayshar $Y$ et al. Cell Stem Cell 2010; 7: 521-531.

10. Baker DE et al. Nat Biotechnol 2007; 25: 207-215.

11. Pasi CE et al. Cell Death Differ 2011; 18: 745-753. 